\title{
A SENSORY NETWORK WITH BINARY TREE TOPOLOGY: PROTOTYPE AND EVALUATION
}

\author{
V. Vengláír , J. Králík ${ }^{* *}$
}

\begin{abstract}
Sensors are widely used in various applications. In order to make the data handling easier, the sensors are being connected into sensor networks. A new sensor network was proposed. It is a wired network with analogue signal transmission. The purpose of this work has been to build a functional prototype, design tests for performance evaluation, execute them and evaluate the results. Based on the measured data, the network was declared functional as a concept. It can be usable in standard or lower precision applications. However, it is not recommended for high precision applications at the time due to accuracy and consistency issues.
\end{abstract}

Keywords: Wired network, BEST BUS, Binary Enhanced Sensor Tree, no communication protocol, measurement.

\section{Introduction}

The use of sensors has been rising. The applications vary and the requirements on the networks with them. The requirements put on a sensory network in a nuclear plant are certainly different from those for a home conditions monitoring system.

It has become popular to use wireless devices with wide connectivity (Wi-Fi, Zigbee, Z-wave, etc.). This raises the comfort of use, but each device has to be an active node in the network, which also raises the price of each device. There is also the question of interference with other wireless signals and the fact that the energy that is emitted by all devices in our surroundings to cover the place with signal rises as well.

A sensory network with binary tree topology (also called BEST - Binary Enhanced Sensor Tree) has been proposed and patented by Věchet and Krejsa (2017). It is a wired network where all addressing is done by physical location of each end device. In the basic form it does not use any communication protocol as all values from the sensors are read as analogue. The main purpose of this network was to propose a low-cost measuring option.

The purpose of this work is to build a prototype of the above-mentioned network and to evaluate how viable option for measuring it is. What follows is a short description of equipment used for the prototype and test designs. At the end, the test results are evaluated.

\section{Materials and Methods}

\subsection{Hardware prototype}

The sensory network with binary tree topology is a wired network. It has four main elements: 1) cable; 2) leaves that are mounted with the sensors; 3) nodes that enable addressing; 4) root that handles all measurements. A description of each element will follow.

\footnotetext{
Ing. Vojtěch Vengláŕ: Institute of Solid Mechanics, Mechatronics and Biomechanics, Faculty of Mechanical Engineering, Brno University of Technology, Technická 2896/2; 616 69, Brno; CZ, Vojtech.Venglar@vutbr.cz

** Ing. Jan Králík: Institute of Solid Mechanics, Mechatronics and Biomechanics, Faculty of Mechanical Engineering, Brno University of Technology, Technická 2896/2; 616 69, Brno; CZ, Jan.Kralik1@vutbr.cz
} 


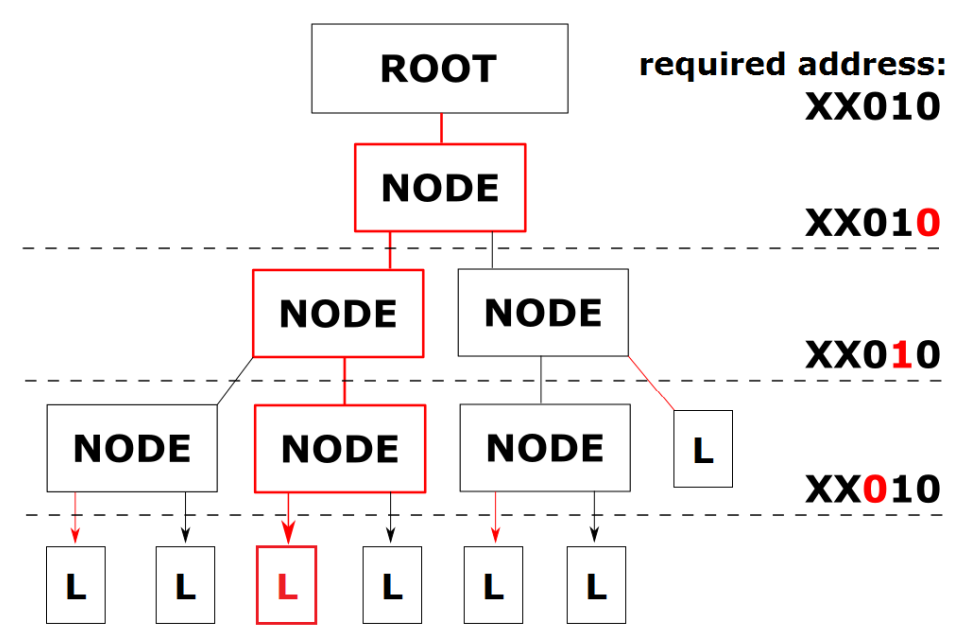

Fig. 1: Sensory network with binary tree topology: block diagram and addressing

Cable defines capabilities of the network as they are determined by wire number. One wire is used as a GND, one as a $5 \mathrm{~V}$ line, one as a data wire. All remaining wires are used as addressing wires. As the network is binary, its size is exponentially dependent on the number of addressing wires ( $\operatorname{size}_{\max }=2^{N}$ ). It also determines the maximum depth of the network (number of layers - see Fig.1). The prototype used a full UTP-Cat5E cable ( 8 wires in 4 twisted pairs) providing 5 address wires. The data wire was in a twisted pair with the GND wire to increase resistance to noise.

Leaf contains a linear voltage stabilizer and one sensor. For the prototype one active and two passive elements were chosen. The passive ones were thermistor (EPCOS, B57871S0123H000) and photoresistor (Silonex, NSL-19M51). They were connected into a voltage divider with resistors of $12 \mathrm{k} \Omega$ and $5 \mathrm{k} \Omega$ respectively. The active element was a humidity sensor (Honeywell HIH-5030-001). The linear stabilizer converts voltage in the network from $5 \mathrm{~V}$ to $3.3 \mathrm{~V}$ to ensure stable voltage. All three types of nodes were built in pairs for comparative purposes.

Node contains a multiplexor. It enables switching between two outputs and thus carries out the address switching. One addressing wire is connected to the multiplexor and drives the switching; the rest goes through the node being only shifted so that the next one drives the switching in the following node. The only signal that gets switched by the multiplexor is the data signal. This way all the leaves are powered all the time and the root does not have to wait for them to power up every time.

Root is an element handling the whole network. It supplies the network with $5 \mathrm{~V}$, requests the address switching and measures the analogue value on data wire using ADC. For the following experiment two roots were used. One as a low-cost solution (STM32 Nucleo F303K8) and one as a validator (National Instruments MyRIO).

\subsection{Experiment description}

The hardware built was a prototype. Therefore, the functionality testing was split into three phases: 1) detection of noise; 2) correct switching; 3) signal comparison. Terms long and short connection will be used. Short connection stands for a leaf being connected by a $0.1 \mathrm{~m}$ cable into a node and that being connected by another $0.1 \mathrm{~m}$ cable into the root. The empty output of the node is connected to a cascade of four other nodes by another $0.1 \mathrm{~m}$ cables. The other leaf measured is connected by a $12 \mathrm{~m}$ cable to the last node in the cascade, putting it as deep as possible in the network. The connection with this leaf is called long connection.

Noise detection test was performed using two adjusted leaves (zero leaves) that connected the signal wire with the GND wire. The leaves were put into short and long connection and measurements were performed. Any signal measured in this measurement was considered noise.

Correct switching test was performed using two leaves with photoresistors. Those were put into different conditions (dark and light). Interventions were included into the measurement to ensure that the values measured were not only noise that was by chance stable (the sensor in the dark was shortly put into light and vice versa). Zero leaves were connected with the unused node outputs for the possibility to correctly detect incorrect switch. Various versions of connection were used. 
Signal comparison test was performed using all three sensor types of leaves separately, always connected into the short and long connection. Unused outputs were again filled with zero leaves. Each time both leaves were subjected to the same conditions at the same time. The signals were then compared and standard deviation was calculated. All measurements were repeated with interchanged sensor leaves.

\section{Results}

The noise detected on the short connection was close to $0 \mathrm{~V}$. Measurement on the long connection showed peaks of up to $0.1 \mathrm{~V}$. The switching test never showed false switching or a signal line that did not react to interventions. The comparison of signals from the same conditions showed that the data differ and using better equipment (MyRIO) generally provided data with lower standard deviation. Nucleo standard deviation was on average higher by $0.03 \mathrm{~V}$ than that of MyRIO.

Tab. 1: Standard deviation of the two compared signals for each type of leaf $[V]$

\begin{tabular}{cccc}
\hline & Photoresistors & Thermistors & Humidity meters \\
\hline Nucleo & 0.11 & 0.08 & 0.10 \\
\hline MyRIO & 0.13 & 0.03 & 0.05 \\
\hline
\end{tabular}
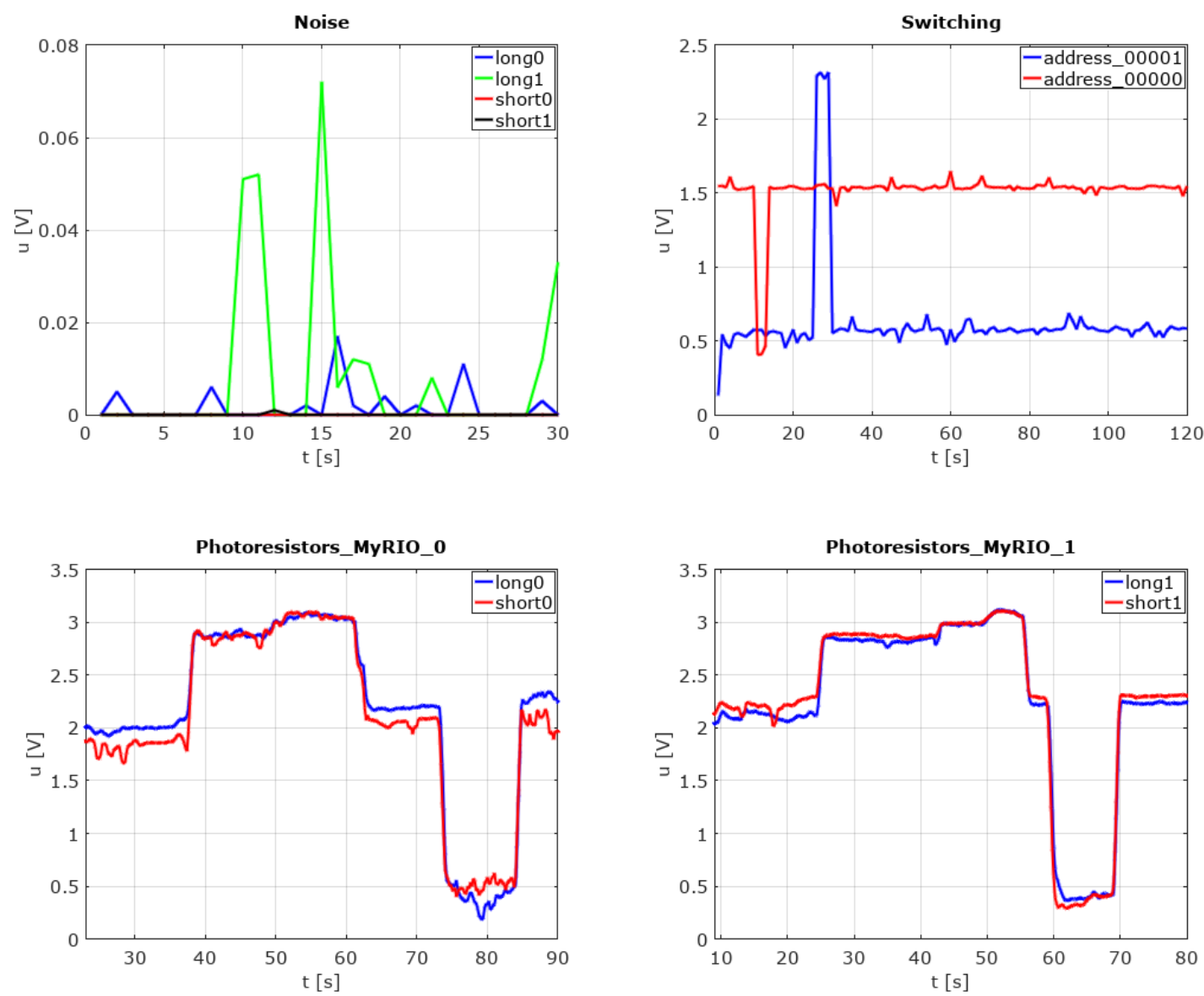

Fig. 2: Plots of measured data from the tests:

Noise: Comparison of noise level on different connection lengths.

Switching: Test of correct node switching.

Photoresistors_MyRIO_0/1: Comparison of signals from sensors on connection of different length. 


\section{Conclusions}

A prototype of sensory network with binary tree topology as proposed by Věchet and Krejsa (2017) was built, a set of tests to evaluate it was designed and the tests were executed. The tests focused on the noise in the network, switching (addressing) capabilities of the network and signal reliability. Measurements were performed using STM32 Nucleo F303K8 (as low-cost possibility) and National Instruments MyRIO (as specialized measurement device for validation).

The noise on the network proved to be too strong for high-precision measurements, but not strong enough to render the network useless. The noise can be removed by filtering (no filter has been applied on the measured data). Further testing showed that the network is capable of proper switching as described in the patent with no detected malfunctions.

As signal reliability indicator the standard deviation between two theoretically identical signals were used. The standard deviation values (Tab.1) and the charts (Fig.2) show that the network is not suitable for highprecision measurements. However, it could be suitable for lower-precision measurements as shapes of the curves were similar, the data only had offsets.

The network proved to be functional. Its main advantage is easy installation, thanks to the binary tree topology, and low cost, as there is no communication protocol that every added sensor would have to handle. There is still room for improvements. The measurements could be done using shielded parts to reduce the noise (cables, connectors).

The network could possibly be used in applications like green house monitoring (Kang et al. (2008)) replacing the wireless network to lower the cost, or home energy management (Věchet et al. (2016)). In a downscaled version it could also be used in mobile robots.

\section{Acknowledgements}

This research was supported by project FSI-S-17-4334 Application of modern methods in modeling, identification and rapid prototyping of control systems for research and development of mechatronic device.

It was also supported directly by Institute of Solid Mechanics, Mechatronics and Biomechanics (Faculty of Mechanical Engineering, Brno University of Technology) by allowing access the National Instruments MyRIO measuring device.

\section{References}

Kang, B.J., Park, D.H., Cho, K.R., Shin, C.S., Cho, S.E., and Park, J.W. (2008) A Study On The Greenhouse Auto Control System Based On Wireless Sensor Network. 2008 International Conference On Security Technology. IEEE, pp 41-44. DOI:10.1109/SecTech.2008.60.

Věchet, S. and Krejsa, J. (2017) A sensory network with the binary tree topology. Patent CZ 306855 B6. issued 2017

Věchet, S., Hrbáček, J. and Krejsa, J. (2016) Environmental Data Analysis for Learning Behavioral Patterns in Smart Homes. In: Proceedings of 17th International Conference on Mechatronics - Mechatronika (ME), IEEE, pp 386-391. 